\section{Brain, Behavior and Evolution}

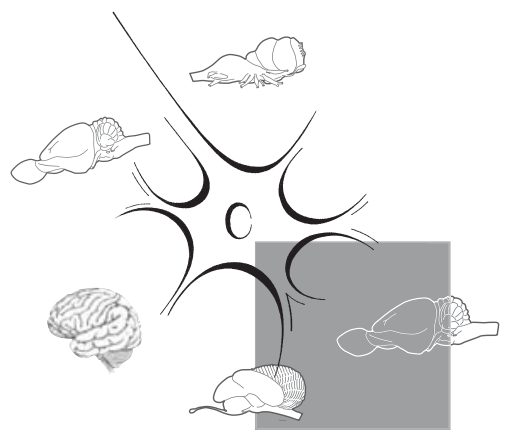

It is widely accepted that there was something exceptional about human cognitive evolution. Among extant primates we are behaviourally and cognitively distinct [Laland and Brown, 2011]. This distinction evolved during our descent from an ape-like ancestor, was shaped by natural selection, and must have a proximate basis in neural adaptation and specialisation. Identifying these adaptations has long been a goal of evolutionary biologists [Striedter, 2005]. An often-cited candidate is the expansion of the frontal regions of the neocortex which, it has been argued, became exceptionally large during human evolution [Rilling, 2006]. Recent work, however, suggests that this was not the case [Barton and Venditti, 2013].

Previous claims for a human-specific enlargement of the frontal lobes have, in fact, been inconsistent [see Rilling, 2006]. Some studies have claimed that there was global expansion of the frontal lobe, whilst others have argued for a specific expansion of particular regions (e.g. the prefrontal cortex), tissue types (e.g. white matter), or hemispheres. Some have shown the pattern was human-specific, others a characteristic of great apes, or even apes in general. Barton and Venditti [2013] argue this ambiguity is a product of using different measures of frontal lobe size, including some that do

\title{
The Human Frontal Lobes: Not Relatively Large but Still Disproportionately Important? A Commentary on Barton and Venditti
}

\author{
Stephen H. Montgomery \\ Department of Genetics, Evolution and Environment, University College London, \\ London, UK
}

not appropriately correct for the pattern of allometry between the frontal lobes and the rest of the brain. The whole neocortex becomes disproportionately large as brain size increases because white matter, which contains nerve fibres connecting different structures, expands much more rapidly than grey matter or neuron number. Although the ballooning of white matter probably reflects size-related constraints on maintaining connectivity, it can produce the appearance that the cortex - or a specific cortical region - is selectively enlarged when viewed as a proportion of total brain size [Barton and Venditti, 2013].

A second problem affecting some previous studies is the failure to acknowledge the shared evolutionary history of living species. Shared ancestry leads to the expectation that more closely related species should resemble each other more than more distant relatives, and renders comparative data non-independent. Using comparative methods that correct for this non-independence, Barton and Venditti [2013] re-visit several datasets to address the issue of allometry and resolve the debate over the size of the human frontal lobes. To do so they examine the relationship between the size of frontal and nonfrontal structures across non-human primates and assess whether humans fall with- in the $95 \%$ confidence limits of these relationships. In contrast to the previous inconsistencies, their results are remarkably unambiguous. Regardless of the region or tissue type used as the focal trait, be it whole frontal cortex, prefrontal cortex, prefrontal white matter, or limbic frontal cortex, humans scale just like any other primate. The same is true when the taxonomic net is spread to apes; they too have frontal lobes that scale like those of other primates.

Perhaps though, there is something peculiar about the rate at which the frontal lobes evolved in humans? Evolutionary rates can be used as an indicator of the strength of selection, so to test this hypothesis, Barton and Venditti apply a model of evolution that estimates the rate of phenotypic evolution along different branches in a phylogeny to identify upwards shifts indicative of changes in selection pressure [Venditti et al., 2011]. But again, there is nothing to indicate that the evolutionary trajectory of humans, or apes, was exceptional. Although the human lineage has the highest rate of evolution for absolute frontal lobe size, when the allometric relationship with other brain structures is accounted for, the human branch is unremarkable.

These combined results imply that the size of the frontal lobes in humans is as

\section{KARGER}

E-Mail karger@karger.com

www.karger.com/bbe
C 2013 S. Karger AG, Base

0006-8977/13/0823-0147\$38.00/0
Stephen H. Montgomery

Department of Genetics, Evolution and Environment

University College London

London WC1E 6BT (UK)

E-Mail stephen.montgomery@ cantab.net 
would be expected for a primate with our brain size. Indeed, a growing number of studies have found a similar conclusion. Beyond volumetric scaling, for example, the human cortex has the same number of neuronal and non-neuronal cells as predicted by their allometric scaling rules in primates [Azevedo et al., 2009]. This conserved volumetric and cellular scaling suggests some underlying constraint, either functional or developmental, which our species adheres to.

Nonetheless, evidence that the human frontal lobes do not depart from allometry, and are therefore no more functionally derived than expected, has previously been met with some opposition, and the debate will most likely rumble on. Several authors [e.g. Deacon, 1990; Passingham, 2002; Striedter, 2005; Dunbar and Shultz, 2006; Rilling, 2006] have noted that even if the human frontal lobes are not relatively large, because they scale hyper-allometrically with the rest of the brain, as they increase in volume they become disproportionately large - that is, they occupy an increasing percentage of the brain's total volume. This fact alone, it is argued, could lead to increased functional capacity without shifts in relative size [Deacon, 1990; Striedter, 2005]. The logic of this hypothesis is that large structures are expected to be more connected to others, and therefore an increase in proportional size may involve changes in the connections the frontal lobe makes with other structures, allowing them to exert a greater influence on other brain regions [Striedter, 2005]. Other authors dismiss this idea on the grounds that it requires special pleading in favour of the frontal lobes, conflates volume with computation capacity when other traits that scale in different ways, such as neuron or synapse number, may be more relevant, requires a greater cognitive output per gram of primate frontal lobe than that of cetaceans or elephants, and fails to dismiss the null hypothesis that shared allometries reflect shared constraints [Barton and Venditti, 2013].

Emphases on either changes in relative size or changes in proportional size as the ecologically or cognitively pertinent trait make different assumptions about how selection and constraints act on brain structure. Unfortunately, these assumptions are rarely discussed. Further confusion is caused by the ease with which proportional and absolute size are conflated, and differing uses of 'adaptation' and 'adaptive change'.
Relative brain size is measured as the departure in the size of a trait from the allometric relationship predicted from related taxa. Brain structures which deviate from allometry have been shown to be associated with various ecological traits [for reviews, see Striedter, 2005; Healy and Rowe, 2007]. A common interpretation of this pattern is that allometry between two structures represents a conserved function or functional constraint [Barton and Harvey, 2000], in which case changes in size without deviation from the predicted allometric pattern are still adaptive because they serve to maintain a vital function. In contrast, deviation from this pattern may reflect a new adaptation, that is, an adaptive change in function. For example, if two correlated structures increase in size, either due to selection on the overall size of the system or one particular component of it, the relative size of each structure will remain constant, as this is necessary to maintain the basic co-functionality between the two structures. If alterations in function are shaped by selection, the relationship between the two structures may change, and this would bring about a change in the relative size of both.

Under the view above, changes in proportional size are also adaptive, as they are products of an allometry imposed by functional constraint, so an increase in proportional size serves to maintain the basic functional relationship, perhaps by maintaining or changing the underlying functional connectivity. However, the change in proportional size would not represent a new adaptation in itself because it is one component of a functionally connected system. In the absence of changes in relative size, for changes in proportional size to represent a new adaptation that was specifically selected for, the underlying constraints must be quite different; the co-evolution between two structures cannot reflect strong functional constraints because the change in size must produce an additional volume of tissue that is not necessary to maintain the ancestral function.

Instead, we could invoke a strong developmental constraint as the cause of the allometric relationship, such that it is easier for selection to bring about changes in the size of suites of structures, rather than individual ones [Finlay and Darlington, 1995]. In this case allometry does not necessarily reflect an adaptive co-functionality. Under this assumption selection on the overall size of the system could produce some functional redundancy in one or more components, particularly in the presence of hyper-allometry. This 'excess' could then become the fodder for secondary selection to act upon and ultimately bring about changes in the substructure, connections or function of one or both components. If selection acts on the volume of a specific structure, then developmental constraints may bring about increased volumes of associated structures which are then reshaped by positive selection or lost by negative selection, for example by targeted apoptosis or changes in the later stages of development. In the case of the latter, which may be more likely if the excess tissue imposes a significant energetic cost, there could also be a secondary change in relative size. In these scenarios, changes in the proportional (or perhaps absolute) size of a structure under direct selection could be regarded as an adaptation, if it is associated with evolutionary changes in function, and may or may not be associated with changes in relative size.

My presentation of these arguments is oversimplified, but it encourages more exploration of the assumptions that underpin different interpretations of the importance of size. For changes in the proportional size of the frontal lobes to reflect an adaptation in the absence of changes in relative size, we would expect there to be weak functional constraints but strong developmental constraints between the frontal lobes and their correlated structures. We could also argue that selection may have been initially acting on the overall size of the system, and that any functional changes in the frontal lobe are the product of secondary selection.

The nature of the constraints acting on brain architecture is a long-running debate. In mammals the relatively conserved developmental timing of brain components and the allometric relationships between them has been interpreted as evidence for substantial developmental constraints [Finlay and Darlington, 1995]. Although this approach does suggest the presence of strong constraints, it does not necessarily demonstrate that they are developmental in nature. Furthermore, there is strong evidence that allometric deviations or shifts in brain structure are possible, both at a coarse [Barton and Harvey, 2000] and finer scale [Barton, 2007]. To identify the nature of genetic/developmental constraints one promising route is the adoption of a quantitative genetics approach. A recent study by Hager et al. 
[2012] found little evidence for genetic correlations between different brain components in lab strains of the house mouse, suggesting the absence of either pleiotropic effects of genetic changes or shared developmental mechanisms between brain components [for commentary, see Vallender, 2013]. Studies in lab mice have garnered criticism as being removed from real fitness effects and previous quantitative genetic studies of brain:body allometry produced varied results in different lab strains [e.g. Atchley et al., 1984; Belknap et al., 1992], but the results of Hager et al. [2012] are in line with quantitative genetic studies in pedigrees of primates. For example, Fears et al. [2009] found that after including total brain volume as a covariate the volume of the cerebrum, cerebellum, hippocampus and corpus callosum all have a significant genetic component suggesting each phenotype is highly heritable independently of total brain size. Intra-specific studies such as these suggest the developmental constraints acting on brain compo- nents are not necessarily sufficiently strong to restrict the action of selection, or drift, from altering allometry. Pinpointing the nature of constraints on a macro-evolutionary scale is challenging, but the fact that co-evolution between brain components closely mirrors the functional connections between them implies some level of functional constraint [Barton and Harvey, 2000].

My own reading of the literature therefore suggests that developmental constraints may be insufficiently strong and functional constraints not sufficiently weak to render likely the scenario necessary for proportional size to be viewed as an adaptation. Of course, others may come to different conclusions. Regardless, when making arguments about different measures of size we must be explicit about the functional and developmental consequences these arguments invoke. To resolve the debate a greater understanding of the nature of the constraints that produce allometric relationships between brain components is clearly needed, alongside further data on the functional consequences of changes in absolute or proportional size. Of course, even when armed with this information, the identification of one particular region of the brain as exceptional requires a full assessment of the functional significance of other correlated structures [Barton, 2012]. In this sense, understanding how selection acts on functionally linked components of distributed neural networks, how these produce complex behaviour, and how selection has acted on different networks in different groups of animals will play a vital role in understanding the evolution of the human brain.

\section{Acknowledgements}

I am grateful to Robert Barton for helpful discussions, and to the Royal Commission for the Exhibition of 1851 for financial support.

\section{References}

Atchley WR, Riska B, Kohn LAP, Plummer AA, Rutledge JJ (1984): A quantitative genetic analysis of brain and body size associations, their origin and ontogeny: data from mice. Evolution 38:1165-1179.

-Azevedo FAC, Carvalho LRB, Grinberg LT, Farfel JM, Ferretti REL, Leite REP, Filho WJ, Lent R, Herculano-Houzel S (2009): Equal numbers of neuronal and nonneuronal cells make the human brain an isometrically scaled-up primate brain. J Comp Neurol 513:532-541.

Barton RA (2007): Evolutionary specialization in mammalian cortical structure. J Evol Biol 20: 1504-1511.

- Barton RA (2012): Embodied cognitive evolution and the cerebellum. Phil Trans R Soc B 367: 2097-2107.

Barton RA, Harvey PH (2000): Mosaic evolution of brain structure in mammals. Nature 405: 1055-1058.

- Barton RA, Venditti C (2013): Human frontal lobes are not relatively large. Proc Natl Acad Sci USA 110:9001-9006.
Belknap JK, Phillips TJ, O’Toole LA (1992): Quantitative trait loci associated with brain weight in the BXD/Ty recombinant inbred mouse strains. Brain Res Bull 29:337-344.

Deacon TW (1990): Rethinking mammalian brain evolution. Amer Zool 30:629-705.

Dunbar RIM, Shultz S (2006): Understanding primate brain evolution. Phil Trans R Soc B 362: 649-658.

Fears SC, Melega WP, Service SK, Lee C, Chen K, $\mathrm{Tu} Z$, Jorgensen MJ, Fairbanks LA, Cantor RM, Freimer NB, Woods RP (2009): Identifying heritable brain phenotypes in an extended pedigree of vervet monkeys. J Neurosci 29: 2867-2875.

Finlay BL, Darlington RB (1995): Linked regularities in the development and evolution of mammalian brains. Science 268:1578-1584.

\footnotetext{
Vallender EJ (2013): How brains are built: genetics and evolution. Brain Behav Evol 81:71-73.

-Venditti C, Meade A, Pagel M (2011): Multiple routes to mammalian diversity. Nature 479 : 393-396.
} 